\title{
A Framework for High Energy Factorisation matched to Parton Showers
}

\section{Marcin Bury}

The Henryk Niewodniczański Institute of Nuclear Physics, Cracow, Poland

E-mail: marcin.bury@ifj.edu.pl

\section{Andreas van Hameren}

The Henryk Niewodniczański Institute of Nuclear Physics, Cracow, Poland

E-mail: hameren@ifj.edu.pl

\section{Hannes Jung}

DESY, Hamburg, Germany

E-mail: hannes.jung@desy.de

\section{Krzysztof Kutak}

The Henryk Niewodniczański Institute of Nuclear Physics, Cracow, Poland E-mail: krzysztof.kutak@ifj.edu.pl

\section{Sebastian Sapeta*}

The Henryk Niewodniczański Institute of Nuclear Physics, Cracow, Poland

E-mail: sebastian.sapeta@ifj.edu.pl

\section{Mirko Serino ${ }^{\dagger}$}

The Henryk Niewodniczański Institute of Nuclear Physics, Cracow, Poland

E-mail: mirko.serino@ifj.edu.pl

High Energy Factorisation was applied so far almost exclusively to Deep Inelastic Scattering, as computing gauge invariant matrix elements with off shell external legs is a highly non trivial task. In recent years, this problem has been completely solved in a variety of ways, both analytically and numerically. The times are mature to produce the first phenomenological predictions. We present the first framework to produce predictions for hadron colliders based on matching off shell gauge invariant matrix elements in the High Energy Factorisation kinematics and for any Standard Model process to the parton showers implemented in the CASCADE program. We then discuss predictions for 4-jet phenomenology, with a special focus on the description the CMS data for inclusive four-jet production with and without Multi Parton Interactions (MPIs).

The European Physical Society Conference on High Energy Physics

5-12 July, 2017

Venice

\footnotetext{
* Speaker.

${ }^{\dagger}$ Speaker.
} 


\section{Introduction}

Using gauge-invariant scattering amplitudes with initial state off-shell particles, we elaborate on our previous studies of 4-jet production and Double Parton Scattering effects [1,2], adding on top of them parton showers with the CASCADE Monte Carlo [3].

The problem of efficiently computing gauge invariant tree level scattering amplitudes in High Energy Factorization (HEF) [4] has been completely solved in recent years, analytically and numerically [5-11].

\section{Outline of previous results in High Energy factorization without parton showers}

The HEF formula for 4-jet production is

$$
\begin{gathered}
\sigma_{4-j e t s}^{B}=\sum_{i, j} \int \frac{d x_{1}}{x_{1}} \frac{d x_{2}}{x_{2}} d^{2} k_{T 1} d^{2} k_{T 2} \mathscr{F}_{i}\left(x_{1}, k_{T 1}, \mu_{F}\right) \mathscr{F}_{j}\left(x_{2}, k_{T 2}, \mu_{F}\right) \\
\times \frac{1}{2 \hat{s}} \prod_{l=1}^{4} \frac{d^{3} k_{l}}{(2 \pi)^{3} 2 E_{l}} \Theta_{4-j e t}(2 \pi)^{4} \delta\left(x_{1} P_{1}+x_{2} P_{2}+\vec{k}_{T 1}+\vec{k}_{T 2}-\sum_{l=1}^{4} k_{l}\right) \overline{\mid\left.\mathscr{M}\left(i^{*}, j^{*} \rightarrow 4 \text { part. }\right)\right|^{2}}
\end{gathered}
$$

Here $\mathscr{F}_{i}\left(x_{k}, k_{T k}, \mu_{F}\right)$ is a transverse momentum dependent (TMD) parton distribution function for a given type of parton; $x_{k}$ are the longitudinal momentum fractions and $\mu_{F}$ is a factorization scale. The new degree of freedom introduced w.r.t. collinear factorization is the transverse momenutm $k_{T k}$, which is perpendicular to the collision axis. The formula is valid when the $x$ 's are not too large and not too small (in the latter case, non linear effects due to saturation and other highmultiplicity phenomena could come into play ) and, in order to construct a full set of TMD parton densities, we apply the Kimber-Martin-Ryskin-Watt prescription [12,13] to the CT10nlo collinear PDF set and employ the running $\alpha_{s}$ coming with it; both the renormalization and factorization scales are set equal to half the transverse energy, i.e. the sum of the final state transverse momenta, $\mu_{F}=\mu_{R}=\frac{\hat{H}_{T}}{2}=\frac{1}{2} \sum_{l=1}^{4} k_{T}^{l}$, working in the $n_{F}=5$ flavour scheme.

On the side of the hard process, $\mathscr{M}\left(i^{*}, j^{*} \rightarrow 4\right.$ part. $)$ is the gauge invariant matrix element for $2 \rightarrow 4$ particle scattering in QCD with two initial off-shell legs. For the purpose of the present investigation, we rely on the numerical Dyson-Schwinger recursion in the KaTie library [14] for its computation.

The Single Parton Scattering (SPS) channel clearly dominates for final states with very high transverse momentum, because it is highly unlikely that two partons from one proton and two from the other one are energetic enough for two hard scatterings to take place, as the well-known behaviour of the PDFs for large momentum fractions suggests. For lower cuts on the final state transverse momenta, a window opens to observe a significant contribution from a higher-twist effect, Double Parton Scattering (DPS); this consists of the simultaneous occurrence of two hard scatterings, each initiated by two partons coming from the first and the second colliding proton respectively. It was analysed in detail, in the context of HEF and for this final state, in our previous works $[1,2]$. 
The standard pocket formula used for the computation of DPS cross sections for a four-parton final state is simply

$$
\frac{d \sigma_{4-j e t, D P S}^{B}}{d \xi_{1} d \xi_{2}}=\frac{m}{\sigma_{e f f}} \sum_{i_{1}, j_{1}, k_{1}, l_{1} ; i_{2}, j_{2}, k_{2}, l_{2}} \frac{d \sigma^{B}\left(i_{1} j_{1} \rightarrow k_{1} l_{1}\right)}{d \xi_{1}} \frac{d \sigma^{B}\left(i_{2} j_{2} \rightarrow k_{2} l_{2}\right)}{d \xi_{2}}
$$

where the $\sigma(a b \rightarrow c d)$ are standard $2 \rightarrow 2$ cross sections, $m$ is a symmetry factor to avoid double counting identical processes, whereas $\xi_{1}$ and $\xi_{2}$ are generic kinematical variables. The effective cross section $\sigma_{e f f}$ is a loose parameterisation of the correlations in transverse space between two partons in the same proton. We use the widely popular value value $\sigma_{e f f}=15 \mathrm{mb}$, on which the large majority of the experimental analysis at the Tevatron and at the LHC agree, within experimental errors. On the other hand, correlations in the longitudinal direction are assumed to be negligible, i.e.

$$
D_{1,2}\left(x_{1}, x_{2}, \mu\right)=f_{1}\left(x_{1}, \mu\right) f_{2}\left(x_{2}, \mu\right) \theta\left(1-x_{1}-x_{2}\right),
$$

where $D_{1,2}\left(x_{1}, x_{2}, \mu\right)$ is the Double Parton Distribution Function and $f_{i}\left(x_{i}, \mu\right)$ are the ordinary PDFs. and the subscripts 1 and 2 distinguish the two generic partons in the same proton.

The findings of $[1,2]$ can be summarised as follows:

- We performed the first full-fledged computation of 4-jet production in HEF, finding that the total cross sections are a bit suppressed w.r.t. the collinear framework [1]. We showed that some variables usually pointed at as potential smoking guns for the search for DPS are actually well described by an SPS-only computation, at least as long as one sticks to the tree level HEF prediction. We elaborate on this below.

- The symmetric cuts imposed in the experimental analysis of [15] are not well suited to maximise the DPS signal expected from the theory, because dijet production beyond pure LO collinear factorisation is perturbatively unstable for symmetric cuts, due to real radiation effects. The latter are present also in LO HEF. As dijet production enters the calculation of the DPS cross section, one possible solution is to perform searches with asymmetric cuts. We proposed one such set in [1].

- We proposed a set of new variables which could perform well for the search of DPS in the 4-jet channel [2].

\section{High Energy factorization plus parton showers for Single Parton Scattering}

There are, at present, two CMS analyses of 4-jet production $[15,16]$.

Ordering the jets in transverse momentum, the cuts used by the first are $p_{T}>50 \mathrm{GeV}$ for the first and second jets, $p_{T}>20 \mathrm{GeV}$ for the third and fourth jets, $|\eta|<4.7$ for the rapidity and $\Delta R>0.5$ for the jet cone radius parameter. For the second the cuts are $p_{T}>20 \mathrm{GeV}$ uniformly for all the 4-jets, $|\eta|<2.1$ for the $b$-tagged and $|\eta|<4.7$ for the non $b$-tagged jets, with $\Delta R>0.3$.

We will set aside the problem of using an analysis with asymmetric cuts in order to maximise the chances to see the DPS contribution predicted by the theory, discussed at length in [1,2], and we will rather focus on the description of a variable which is supposed to be a smoking gun for DPS 
and which, among the three variables first proposed in [15] to this purpose, is not satisfactorily described by any collinear Monte Carlo. Geometrically, it is the angle between the sum of the momenta of the two hardest and the two softest jets in the final state.

$$
\Delta S \equiv \arccos \left(\frac{\vec{p}_{T}\left(j_{1}^{\text {hard }}, j_{2}^{\text {hard }}\right) \cdot \vec{p}_{T}\left(j_{1}^{\text {soft }}, j_{2}^{\text {soft }}\right)}{\left|\vec{p}_{T}\left(j_{1}^{\text {hard }}, j_{2}^{\text {hard }}\right)\right| \cdot\left|\vec{p}_{T}\left(j_{1}^{\text {soft }}, j_{2}^{\text {soft }}\right)\right|}\right)
$$

The best improvement which can be brought into our framework in order to push it beyond the pure tree level prediction and without inserting loop contributions ( because they are, for now, too demanding for the present level of development of HEF ), is to add parton showers on top of the matrix-element calculation. In order to suit our $k_{T}$-dependent framework, it is desirable to perform a backward evolution of the initial state which can trace back the origin of the transverse momentum of the initial state partons entering the hard matrix to the series of emissions occurred before the hard collision takes place. Without saturation effects and for low values of $x$, too low to be met by the data we are confronting, the BFKL equation would be suitable [18].

An evolution evolution which is built specifically to interpolate between BFKL and DGLAP is the CCFM equation [19]. The CCFM evolution is already implemented in the CASCADE Monte Carlo program [3]. However, CCFM is available at the moment only for gluons, although efforts to extend the framework are in progress [20,21]. For the sake of the present work, we thus choose to use a full-flavour DGLAP evolution, which we match to our parton-level generator in order to preliminary assess the effect of parton showers. Also, we choose to focus for the moment on the pure SPS contribution, as adding parton showers on top of DPS events is more laborious, due to the potential interplay of the showerings from the two scatterings. Our preliminary findings are shown in fig. (1). We observe that, after the showering and including a full remnant treatment, the
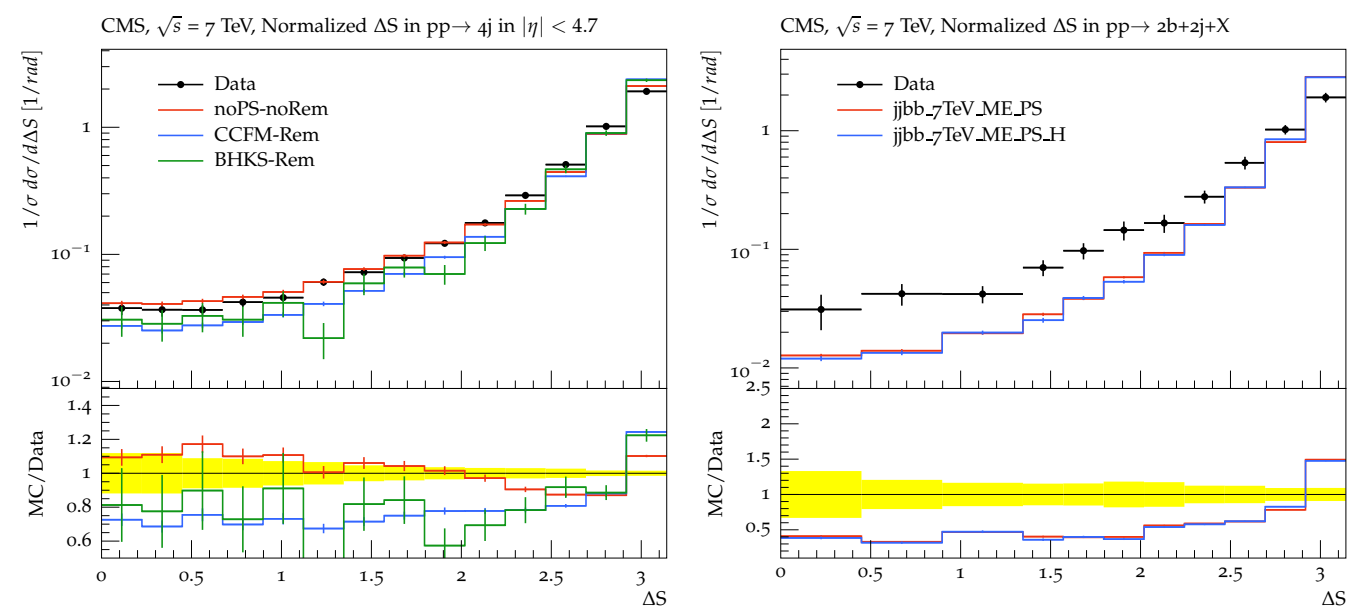

Figure 1: After applying parton showering with full remnant treatment, the pure SPS predictions undershoots considerably the data in the low $\Delta S$ region. The mismatch without DPS seen already for 4 jets is even more apparent when two of them are b-tagged.

SPS prediction is below the experimental data and even more so in the case with two $b$-tagged jets. The predictions shown here have been tested for dependence on the underlying collinear PDF sets, 
employing three more sets and such a dependence was found to be safely negligible. Further study is in progress.

\section{Conclusions}

We provided the first-ever description of 4-jet production in a consistent leading order High Energy Factorization framework missing no contribution and supplemented by parton showers. This seems to point to the need of Double Parton Scattering to explain the CMS data on 4-jet production with and without a pair of $b$-tagged jets. In the latter case, this need is more apparent, in agreement with the experimental findings.

\section{Acknowledgments}

This work is partially supported by the Narodowe Centrum Nauki with Sonata Bis grant DEC2013/10/E/ST2/00656.

\section{References}

[1] K. Kutak, R. Maciula, M. Serino, A. Szczurek, and A. van Hameren, JHEP 04, 175 (2016), arXiv:1602.06814.

[2] K. Kutak, R. Maciula, M. Serino, A. Szczurek, and A. van Hameren, Phys. Rev. D94, 014019 (2016), arXiv:1605.08240.

[3] H. Jung, Comput. Phys. Commun. 143, 100 (2002), arXiv:hep-ph/0109102.

[4] S. Catani and F. Hautmann, Nucl. Phys. B427, 475 (1994), arXiv:hep-ph/9405388.

[5] A. van Hameren, P. Kotko, and K. Kutak, JHEP 12, 029 (2012), arXiv:1207.3332.

[6] A. van Hameren, P. Kotko, and K. Kutak, JHEP 01, 078 (2013), arXiv:1211.0961.

[7] A. van Hameren, K. Kutak, and T. Salwa, Phys. Lett. B727, 226 (2013), arXiv:1308.2861.

[8] A. van Hameren, JHEP 07, 138 (2014), arXiv:1404.7818.

[9] M. Bury and A. van Hameren, Comput. Phys. Commun. 196, 592 (2015), arXiv:1503.08612.

[10] A. van Hameren and M. Serino, JHEP 07, 010 (2015), arXiv:1504.00315.

[11] K. Kutak, A. van Hameren, and M. Serino, JHEP 02, 009 (2017), arXiv:1611.04380.

[12] M. A. Kimber, A. D. Martin, and M. G. Ryskin, Phys. Rev. D63, 114027 (2001), arXiv:hep-ph/0101348.

[13] A. D. Martin, M. G. Ryskin, and G. Watt, Eur. Phys. J. C66, 163 (2010), arXiv:0909.5529.

[14] A. van Hameren, (2016), arXiv:1611.00680.

[15] CMS, S. Chatrchyan et al., Phys. Rev. D89, 092010 (2014), arXiv:1312.6440.

[16] CMS, V. Khachatryan et al., Phys. Rev. D94, 112005 (2016), arXiv:1609.03489.

[17] G. Altarelli and G. Parisi, Nucl. Phys. B126, 298 (1977).

[18] I. I. Balitsky and L. N. Lipatov, Sov.J.Nucl.Phys. 28, 822 (1978). 
[19] M. Ciafaloni, Nucl. Phys. B296, 49 (1988).

[20] O. Gituliar, M. Hentschinski, and K. Kutak, (2015), arXiv:1511.08439.

[21] M. Hentschinski, A. Kusina, and K. Kutak, Phys. Rev. D94, 114013 (2016), arXiv:1607.01507. 